\title{
Study of an Inductive Sensor for Measuring High Frequency Current Pulses
}

\author{
M. Rojas*, G. Robles*, B. Tellini ${ }^{\dagger}$, C. Zappacosta ${ }^{\dagger}$, J.M. Martínez* and J. Sanz* \\ * Departamento de Ingeniería Eléctrica \\ Universidad Carlos III de Madrid, Leganés - 28911 Madrid (Spain) \\ Email:.mvrojas@ing.uc3m.es, grobles@ing.uc3m.es, jmmtarif@ing.uc3m.es, jsanz@ing.uc3m.es \\ ${ }^{\dagger}$ Dipartimento di Sistemi Elettrici e Automazione \\ Università di Pisa 56122 Pisa (Italy) \\ Email: bernardo.tellini@dsea.unipi.it,carmine.zappacosta@dsea.unipi.it
}

\begin{abstract}
The objective of this paper is to present a simple sensor based on Faraday's Law together with an integration and amplifying stage and used for measuring fast current pulses. Its behavior in time and frequency is tested by measuring Partial Discharges (PD) in electric insulation systems following the guidelines given in the standard IEC 60270. The measurements are also taken with two other sensors, a commercial high frequency current transformer and a resistive transducer, with the aim of comparing the results obtained with the proposed probe and obtaining an estimation of its sensitivity. An estimation of the uncertainty of the measurements is also calculated obtaining an error similar to other commercial devices.
\end{abstract}

\section{INTRODUCTION}

In many scientific and technical fields, it is often necessary to determine the amplitude and waveforms of rapidly varying currents. Some of the phenomena related to high frequency currents, and hence, candidates to be measured with the proposed sensor, are lightning discharges, electrical arcs and post-arc phenomenon studies with circuit breakers, electric discharge studies in plasma physics, electrostatic discharge and partial discharges [1], 2]. The pulse rise times can vary from a few nanoseconds to a few microseconds. In all such cases, the sensing devices should be capable of measuring the signal over a wide frequency band involving the use of expensive and sophisticated high-frequency equipment [3. This paper presents a simple inductive sensor together with an integrator as a feasible and inexpensive alternative to other sensors such as high frequency current transformers (HFCT). It can even become a reference transducer for other probes when measuring high frequency currents because of its wide bandwidth and ease of use.

The phenomena used for testing the proposed sensor are partial discharges (PD), a well-known ageing mechanism in dielectrics under high-voltage conditions. PD cause fast current pulses which have a rise time of a few nanoseconds and flow through an external circuit. This circuit is composed mainly of capacitive components (coupling capacitor) where current pulses are detected [4, so they have bandwidths between the units and tens of $\mathrm{MHz}$ [5] which

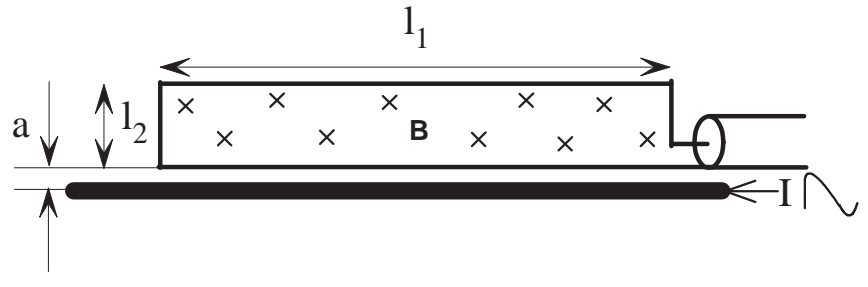

Fig. 1: Inductive sensor schematic.

are in the range of measurement of the proposed sensor. These pulses occur in small cavities or voids within the electrical insulation [6], producing chemical reactions that lead to oxidation and ion bombardment, causing pitting in the walls of the inclusions and, gradually, the breakdown of the insulation. As this activity is considered a serious threat to the life of the insulation, their measurement is of the utmost importance [7, [8, 9].

To detect PD pulses, a setup based on the guidelines given in the standard IEC 60270 [10 is used together with a high frequency current transformer to compare results with a commercial transducer and then with a high frequency resistance to estimate the current value and sensitivity of the proposed probe.

\section{Modeling of the Sensor}

The prototype of the inductive sensor consists of a conductor in the shape of a rectangular loop that is located close to a primary conductor, Fig. 11 11. The current that flows in the primary produces a magnetic flux that links the loop and creates an induced voltage proportional to the time rate of change of the current; integrating this voltage would give a measure of the current.

The frequency content on a pulse derived from a partial discharge depends on many factors, including the type of $\mathrm{PD}$, the insulation where it occurs and the discharge circuit. In most cases, commercial HFCT commonly used in PD detection measure up to $50 \mathrm{MHz}$. The modeling of the sensor is made with a lumped parameters approach in accordance to the maximum frequency contained in these pulses. This imposes a constraint on the length of 
TABLE I: Dimensional parameters for the probe

\begin{tabular}{|c|c|c|c|c|}
\hline \multicolumn{5}{|c|}{ Dimensions $[\mathrm{mm}]$} \\
\hline$l_{1}$ & $l_{2}$ & $h$ & $d$ & $a$ \\
\hline 120 & 9 & 0.381 & 0.050 & 1.016 \\
\hline
\end{tabular}

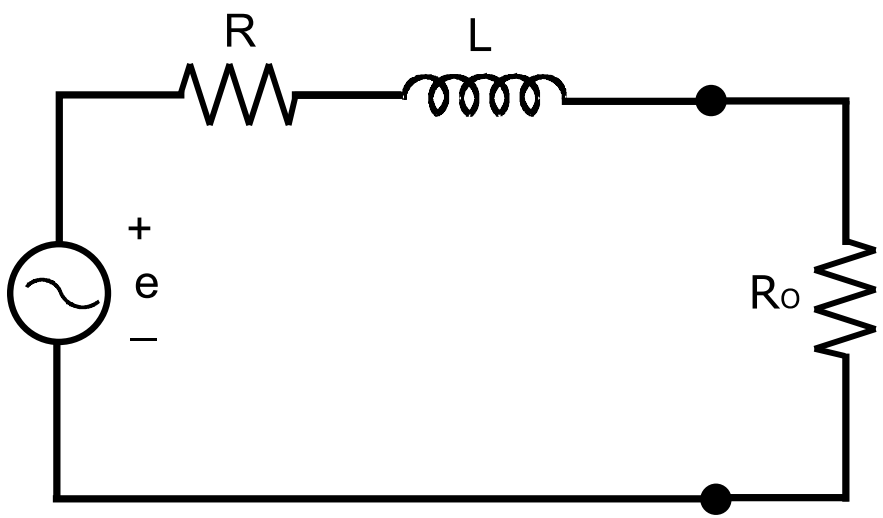

Fig. 2: Electric equivalent circuit of the rectangular loop and the equivalent impedance of the measuring circuit.

sensor 12 to prevent reflections, setting the dimensional parameters of the prototype as those specified in Table I. where $l_{1}$ and $l_{2}$ are the lengths of the rectangular loop, $h$ and $d$ the width and thickness of the conductor of the sensor and $a$ the distance between the loop and the primary conductor.

Fig. 2 shows the equivalent circuit of the probe. The source voltage represents the induced voltage in series with the self-inductance of the loop and the resistance of the conductor that forms the rectangular loop [13].

In this circuit, the induced voltage $e$ is determined by (1).

$$
e=M \cdot \frac{d i}{d t}
$$

Where $M$ is the mutual inductance between the loop and the primary conductor and $i$ is the current pulse. Its equation can be easily derived yielding (2).

$$
M=\frac{\mu_{0}}{2 \pi} l_{1} \ln \frac{l_{2}+a}{a}=54.9 \quad \mathrm{nH}
$$

The value of the self-inductance is shown in (3), deduced from the equation for a rectangle of round wire proposed by Grover in 14 with dimensions in $\mathrm{cm}$. The parameter $\ln g$ is tabulated in [14 according to the relation $d / h$. Substituting the geometric parameters, the inductance is $229.4 \mathrm{nH}$.

$$
\begin{array}{r}
L=0.004\left[l_{1} \ln \left(\frac{2 l_{1}}{h+d}\right)+l_{2} \ln \left(\frac{2 l_{2}}{h+d}\right)-\cdots\right. \\
\cdots\left(l_{1}+l_{2}\right) 0.5-\left(l_{1}+l_{2}\right) \ln g+2 \sqrt{l_{1}^{2}+l_{2}^{2}}-\cdots \\
\left.\cdots l_{1} \sinh ^{-1} \frac{l_{1}}{l_{2}}-l_{2} \sinh ^{-1} \frac{l_{2}}{l_{1}}\right]
\end{array}
$$

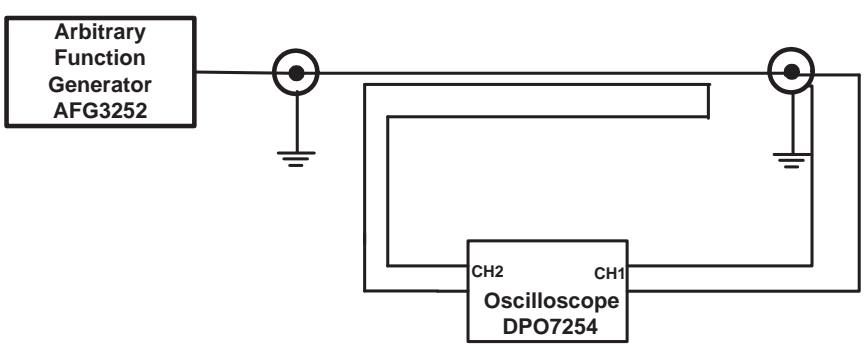

Fig. 3: Experimental setup for the characterization of the sensor.

The resistive value of the sensor model is obtained taking into account the skin effect in (4), as suggested in [11.

$$
R=\frac{l}{2 h} R_{s} \frac{1-e^{-2 d / \delta}-4 e^{-d / \delta} \cos (d / 2 \delta) \sin (d / 2 \delta)}{e^{-2 d / \delta}-2 e^{-d / \delta} \cos (d / \delta)+1}
$$

with

$$
R_{s}=\frac{1}{\delta \sigma}
$$

$R_{s}$ being the skin resistance, $\sigma$ the conductivity of copper, $\delta$ the skin depth and $l$ the total length of the rectangular loop.

It is interesting to study the frequency behavior of the loop to assure that it will measure high frequency currents and, particularly, PD. If the sensor were able to work in open circuit, the output voltage would be the derivative of the primary current at every frequency, giving a steady increase of $20 \mathrm{~dB}$ per decade in the frequency response. However, this is not possible and there has to be a measuring circuit at the output with a finite input impedance modifying the stand-alone frequency behavior. Fig. (2) shows a typical input impedance of an oscilloscope with $R_{o}=50 \Omega$ considering coaxial cables as short as possible so the effect of the connections is negligible. The voltage acquired by the measuring system, $V_{o}$, is the voltage divider given by $L$ on one side and $R_{o}$ on the other side, assuming that $R$ is negligible $(0.58 \Omega$ at $50 \mathrm{MHz})$. The resulting frequency response between the measured voltage and the primary current is a transfer function with a simple pole and a zero in the origin, (6).

$$
\frac{V_{o}(s)}{I(s)}=\frac{M s}{1+L s / R_{o}}
$$

The measuring circuit places the pole at $34.7 \mathrm{MHz}$, which flattens the frequency response from $+20 \mathrm{~dB}$ per decade to $0 \mathrm{~dB}$ per decade so the sensor works as a derivator up to this frequency. An empirical characterization of the frequency response of the inductive sensor was done with the setup shown in Fig. 3 It consists of two circuits: the first one is an arbitrary function generator Tektronix AFG 3252 connected to channel one in an oscilloscope through the primary conductor of the prototype, the input impedance of the oscilloscope serving as a load to the waveform generator; in the second one, the probe is connected to channel two. The generator is set to a sinusoidal signal 

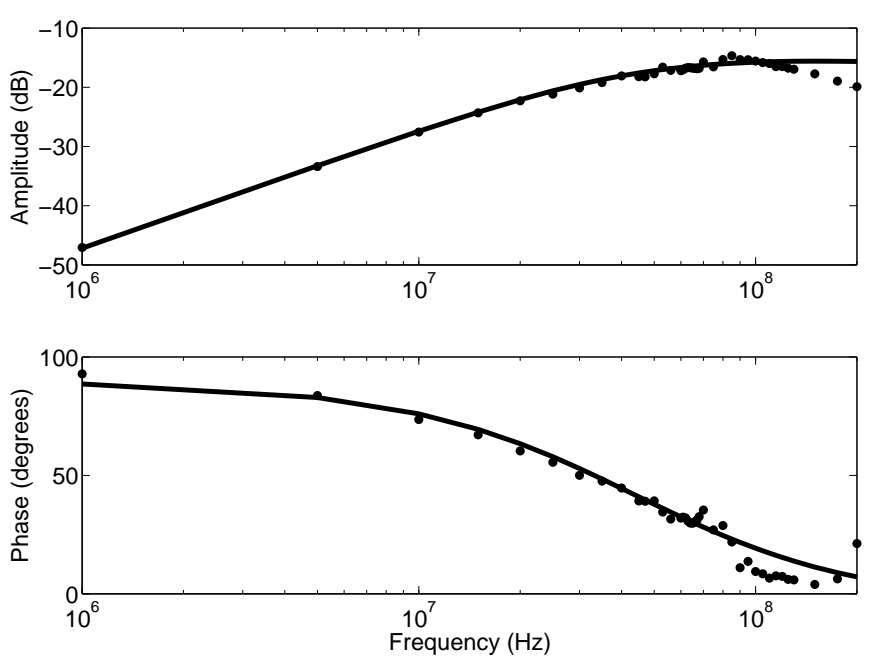

Fig. 4: The frequency response of the inductive sensor. The experimental data(dotted plot) and the theoretical data (solid data).

with an amplitude of $2.5 \mathrm{~V}$ and sweeps a frequency range from $1 \mathrm{MHz}$ to $200 \mathrm{MHz}$. The empirical transfer function should follow (6) so, in the experiment, the input signal is the primary current and the output is the voltage of the probe measured by the oscilloscope. The setup in Fig. 3 is designed to be as simple as possible so there is not a direct measure of the primary current, but it can be calculated as the voltage in channel $1, V_{1}$, divided into the input impedance of the oscilloscope, $I(s)=V_{1}(s) / R_{o}$.

The measurements are shown in Fig. 4 where the solid line is the analytical transfer function in (6) and the dotted line is the empirical transfer function. Both plots are the same up to $110 \mathrm{MHz}$; for higher frequencies, the effect of the cables should be considered because they introduce a new pole that drops the gain with a rate of $-20 \mathrm{~dB}$ per decade. Nevertheless, the results confirm that there is a pole at $34.7 \mathrm{MHz}$ limiting the band of the inductive sensor, as predicted with the equivalent circuit. Section IV will show that with an integration stage connected directly to the loop, the bandwidth can be extended beyond this frequency and the output signal will be directly proportional to the current pulse.

\section{Measurements}

This Section shows the behavior of the probe in a standalone configuration - without an integration stage - to demonstrate that it can still measure high frequency currents and opens the possibility of acquiring the pulses with a digitizing system to integrate them. These pulses were produced with the setup shown in Fig. 5 following the guidelines for measuring $\mathrm{PD}$ given in the standard IEC 60270 [10]. A high voltage transformer was connected to the device under test (DUT), an electrode on a polymeric sheet connected to ground, where surface PD occur. A capacitive divider was connected in parallel to the DUT to provide a path for the current generated by the PD in the sample. The designed sensor was placed together with

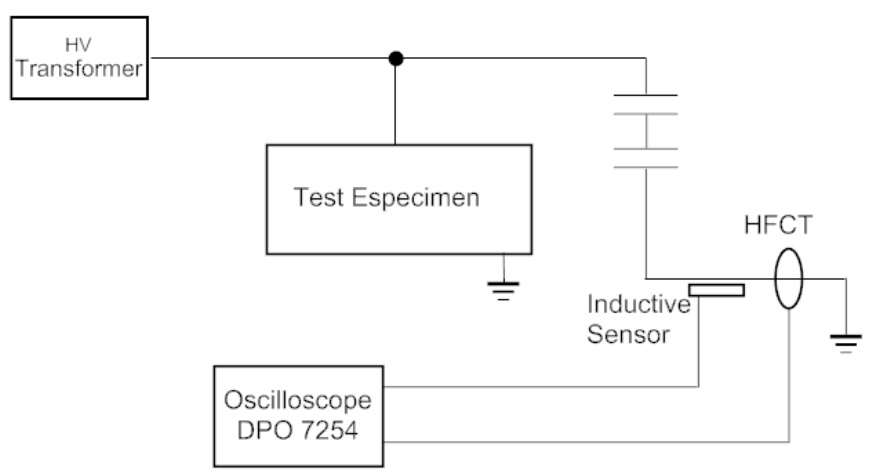

Fig. 5: Experimental setup.

an HFCT, so that we could have a reference of the pulse shape from a commercial sensor. However, it is not used as a current amplitude reference because the V/A sensitivity given by the transformer manufacturer is different from what we obtain in our setups and the frequency response is unknown. The transducers were connected at the input of the oscilloscope to measure and record the pulse amplitude and shape.

The setup is based on a simple test object at a fixed voltage of $2000 \mathrm{~V}$ so that $\mathrm{PD}$ activity can be easily controlled, obtaining pulses with the same shape [15]. Fig. 6 shows a screen capture of the oscilloscope where the grey plot is the signal from the HFCT and the black one the pulse obtained with the inductive sensor. The sampling frequency is $10 \mathrm{GS} / \mathrm{s}$, the time per division is $50 \mathrm{~ns}$ and the vertical scales per division are $100 \mathrm{mV}$ and $50 \mathrm{mV}$ for the HFCT and the sensor, respectively. This plot shows a raw signal obtained with the inductive sensor that, without additional conditioning, already has a peak at $80 \mathrm{mV}$. Notice that the pulse obtained with the sensor is the derivative of the HFCT signal, where the maxima of the HFCT pulse correspond to zero crossings in the sensor pulse.

To get a signal proportional to the current pulse, a numerical integration of the sensor pulse was done in Matlab and the results are shown in Fig. 7 The upper plot is the signal from the HFCT and the lower one the integrated pulse of the inductive sensor.

Furthermore, we can observe that the duration of the signals is very short, in the order of hundreds of nanoseconds. Thus, the inductive sensor has a suitable response equal to HFCT for measuring high frequency signals.

Another way to get a signal proportional to the current pulse in real time is the design and implementation of an integration stage in the sensor. This will be explained in the next section.

\section{IMPLEMENTATION OF AN INTEGRATOR}

\section{A. Design of the prototype}

After several tests with different operational amplifiers and differential amplifiers, the best results were obtained with the integrator shown in Fig. 8 using a pair of VCA 824 amplifiers. This differential amplifier was finally chosen 


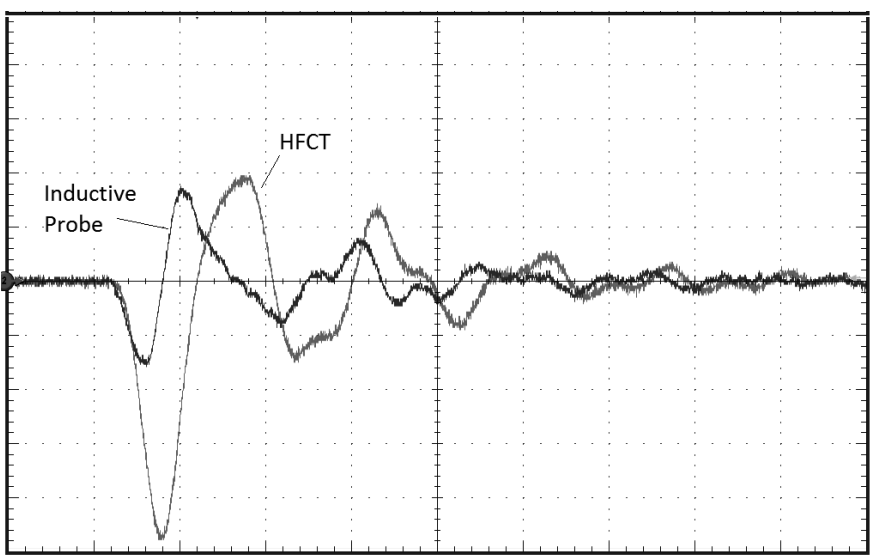

Fig. 6: PD pulse detected with the inductive sensor (smaller plot) and the Bergoz HFCT (larger plot) at 2000 $\mathrm{V}$ in the polymer sheet. The vertical scales per division are $100 \mathrm{mV}$ and $50 \mathrm{mV}$ for the HFCT and the sensor, respectively, and the time per division is $50 \mathrm{~ns}$.
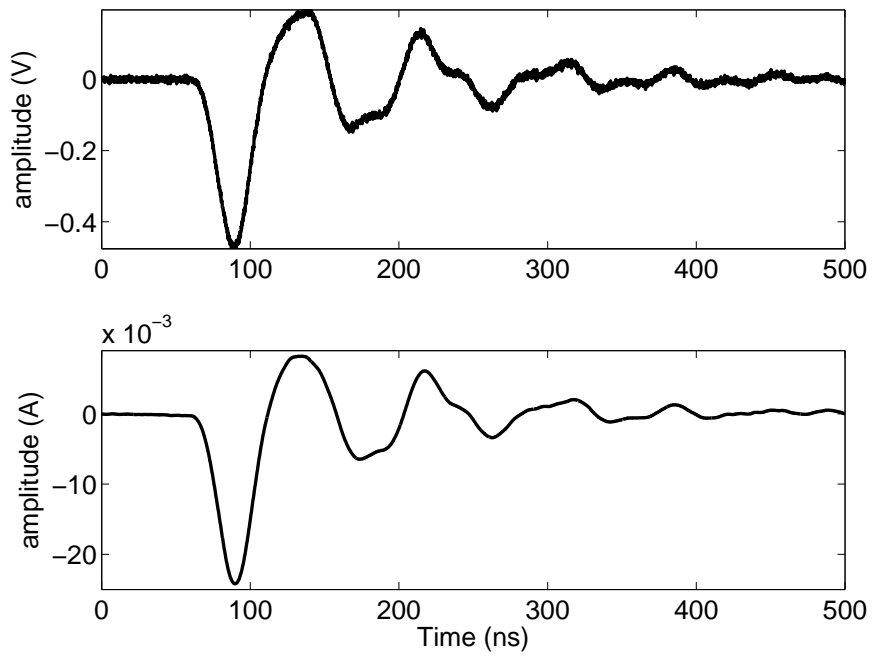

Fig. 7: Current pulse that corresponds to a PD at 2000 $\mathrm{V}$ in the polymer sheet measured with the Bergoz HFCT (upper plot) and the numerically integrated pulse from the inductive probe (lower plot).

because it has a high input impedance and a low input capacitance, $1 \mathrm{M} \Omega$ and $1 \mathrm{pF}$, respectively; the bandwidth suited our needs, reaching $420 \mathrm{MHz}$, and it has a low input noise of $6 \mathrm{nV} / \sqrt{\mathrm{Hz}}$. The circuit is detailed in Fig. 9 and the block diagram is showed in Fig. 10. The prototype consists of three stages, a differential amplification, a passive integration and a second amplification. The first stage has a high input impedance, $R_{S 1}=R_{S 2}=1 \mathrm{k} \Omega$, so as not to charge the inductive loop. This stage also maintains the phase of the original signal with an equalizing RC network at the input as required in [16]. The transfer function has a zero at $R_{g 1} C_{2}$ that compensates the pole of the integration block in the next stage, and a pole at $R_{1} C_{2}$ that extends the bandwidth. The gain is set to $10 \mathrm{~V} / \mathrm{V}$ with the resistors $R_{g 1}$ and $R_{F 1}$ and then adjusted with a variable resistor connected to pin $V_{G}$, not shown in Fig. 9 for the sake of

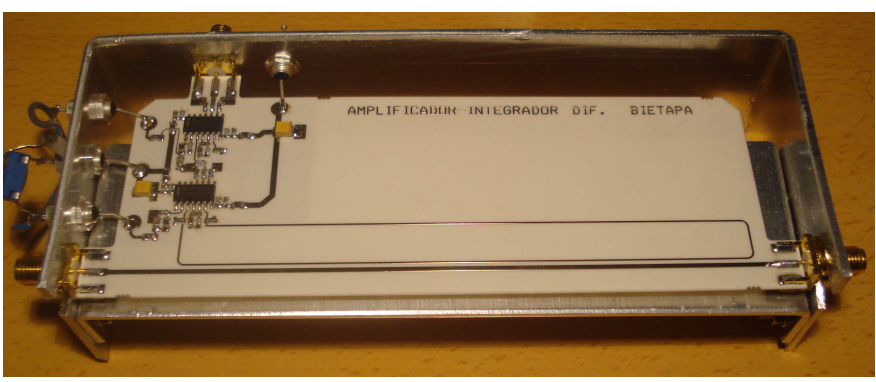

Fig. 8: Picture of the integrator prototype.

clearness in the schematic. The gain can vary from 0.1 $\mathrm{V} / \mathrm{V}$ to $10 \mathrm{~V} / \mathrm{V}$ when the voltage at $V_{G}$ varies from -1 $\mathrm{V}$ to $1 \mathrm{~V}$, respectively. In the prototype, the gain was set to a compromise value to assure the best bandwidth and equalization.

The second stage is another $\mathrm{RC}$ network, with $620 \Omega$ and $2200 \mathrm{pF}$, that places a pole near $100 \mathrm{kHz}$ and integrates above $1 \mathrm{MHz}$.

The final stage is complemented with another VCA 824 amplifier to boost the total gain of the circuit. The voltage at the positive input is the output of the integration network and a matched resistance of $620 \Omega$ to ground is connected to the negative input so the VCA 824 is as a mere amplifier. Capacitance $C_{13}$ and resistance $R_{L 1}$ place a pole at low frequencies to block the DC component of the first stage and possible external $50 \mathrm{~Hz}$ noise.

It is necessary take into account that due to the derivative condition of the loop, the amplitude of signal increases as the frequency does. Thus, for frequencies above $60 \mathrm{MHz}$ and for pulses of high amplitude, the output signal can be affected by saturation. Considering this limitation, an empirical characterization of the transfer function of the integrator prototype was done based on the setup shown in Fig 3. The generator is set to a sinusoidal signal of 0.25 $\mathrm{V}$ of amplitude and sweeps a frequency range from $1 \mathrm{MHz}$ to $60 \mathrm{MHz}$.

The output signal is the voltage at the output of the integrator prototype and the input signal is the voltage in channel 1 divided by $50 \Omega$ to obtain the current. The experimental frequency response is shown in Fig. 11.

The upper plot is the relation between amplitudes, which is flat at $6 \mathrm{~dB}$ up to $50 \mathrm{MHz}$, and the lower plot is the phase shift between the signals; notice that both signals are in phase in the same range of frequencies. The slight rise in the gain plot is due to the interaction of the self-inductance of the loop and the capacitance of the equalizing network; these give a resonance frequency in $104 \mathrm{MHz}$ that modifies the flatness of the response. Thus, we can conclude that the bandwidth of the final prototype with integration is defined between $1 \mathrm{MHz}$ and $50 \mathrm{MHz}$, which is adequate for measuring high frequency pulses such as PD [17]. 


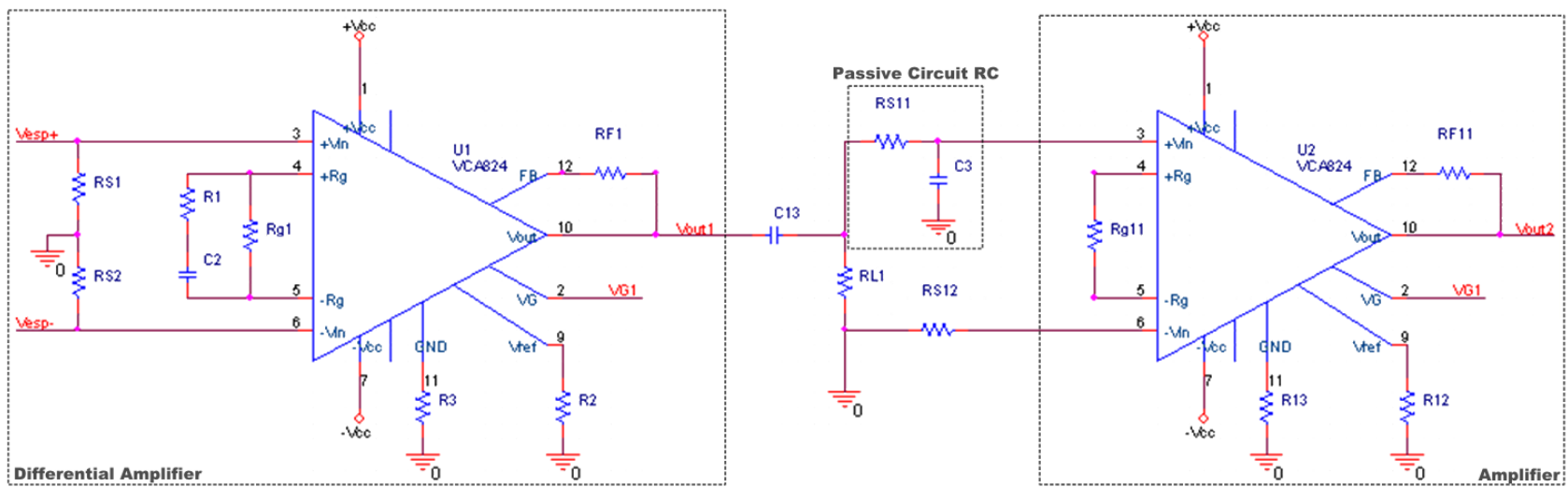

Fig. 9: Schematic of the integrator stage.

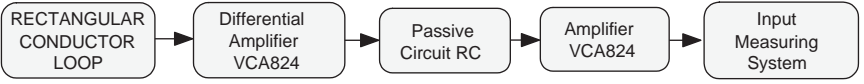

Fig. 10: Block diagram of the integrator prototype.
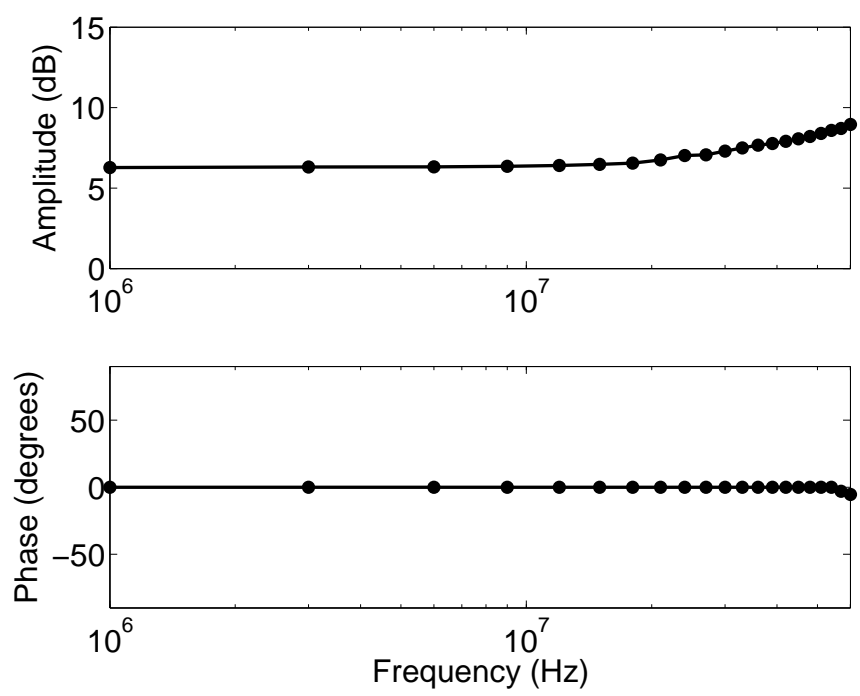

Fig. 11: Experimental response of the integrator prototype between 1 and $60 \mathrm{MHz}$.

\section{B. Measurements}

The experimental validation was done by applying 2000 $\mathrm{V}$ between an electrode and ground separated by a polymeric sheet. The measurements were made with an HFCT as a commercial reference for the pulse shape and then with a high frequency resistance with the aim of estimating the current value and the prototype sensitivity.

Figs. 12 and 13 represent two examples of pulses of different polarity obtained simultaneously with the final prototype and the HFCT. In this case, the sampling frequency is $2.5 \mathrm{GS} / \mathrm{s}$, the time per division is $100 \mathrm{~ns}$ and the vertical scales per division are $300 \mathrm{mV}$ and $80 \mathrm{mV}$ for the HFCT and the final prototype, respectively.

Again, the results are satisfactory since the final prototype signal follows the HFCT signal reliably, verifying

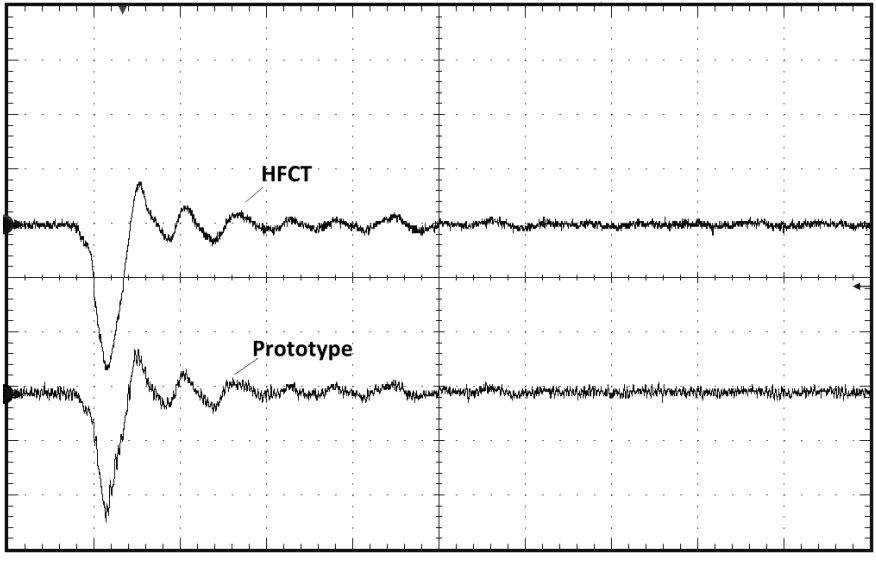

Fig. 12: Negative current pulse that corresponds to a PD at $2000 \mathrm{~V}$ in the polymer sheet measured with the Bergoz HFCT (upper plot) and the integrator prototype (lower plot). The vertical scales per division are $300 \mathrm{mV}$ and 80 $\mathrm{mV}$ for the HFCT and the final prototype, respectively, and the time per division is $100 \mathrm{~ns}$.

that the proposed sensor has the capacity to measure $\mathrm{PD}$ pulses similarly to a commercial transducer. A quantitative measurement of the likelihood of the two signals is given by calculating the correlation coefficient of the two series [18. In the case of the examples, the correlation coefficients are 0.96 and 0.98 for the negative and positive pulses, respectively [19.

The next step was to repeat the pulse measurements to estimate the amplitude of the current using a resistance as a reference. The chosen resistor has a flat response in amplitude and phase up to $1 \mathrm{GHz}$ and a value equal to $50 \Omega$ [20. This resistance was located in the capacitive branch of the experimental setup instead of the HFCT, as shown in Fig 14 Both sensors, the resistance and the final prototype, are connected to the $50 \Omega$ input of the oscilloscope so, in the case of the resistance, the measured current is divided into two halves. One half flows through the resistive transducer and the other half through the input resistance of the oscilloscope.

This resistance gives a good signal and it is an inexpen- 


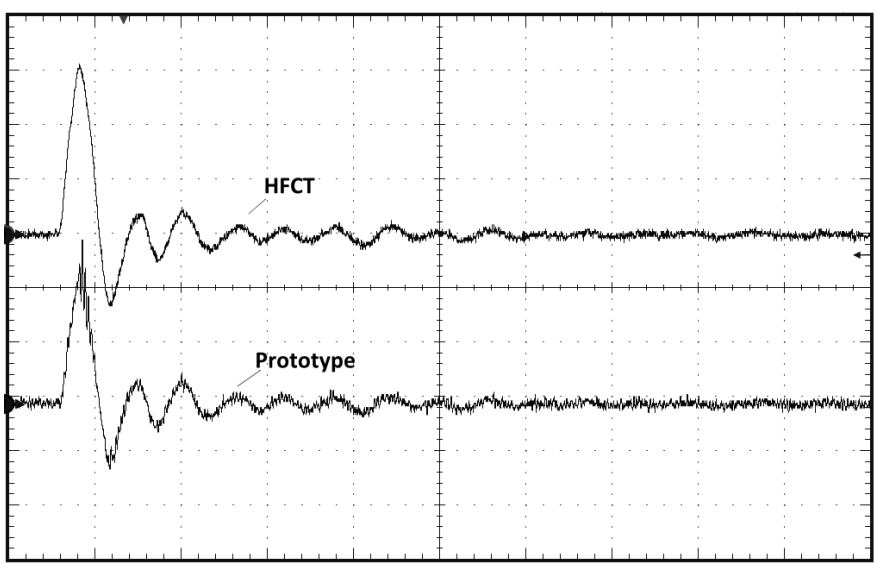

Fig. 13: Positive current pulse that corresponds to a $\mathrm{PD}$ at $2000 \mathrm{~V}$ in the polymer sheet measured with the Bergoz HFCT (upper plot) and the integrator prototype (lower plot). The vertical scales per division are $300 \mathrm{mV}$ and 80 $\mathrm{mV}$ for the HFCT and the final prototype, respectively, and the time per division is $100 \mathrm{~ns}$.

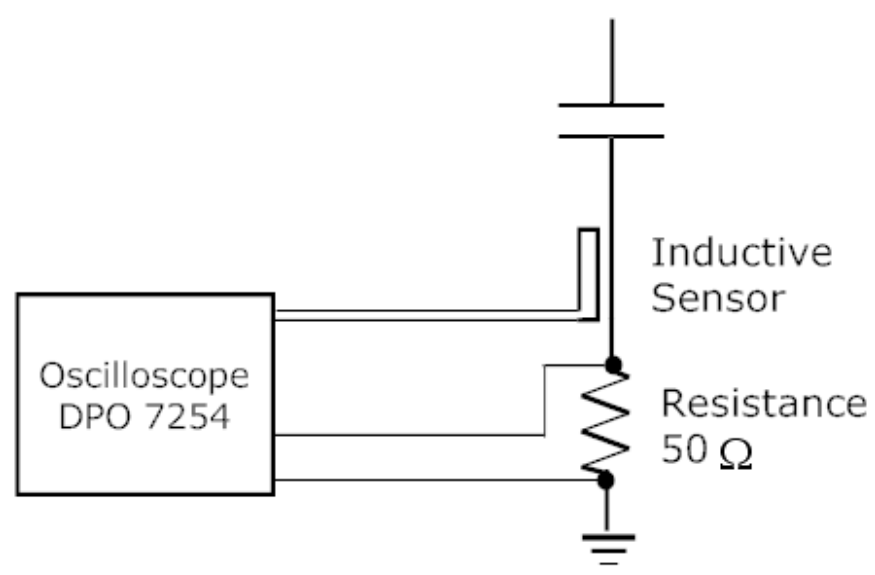

Fig. 14: Capacitive branch of the experimental setup in Fig. 5 with the resistance.

sive sensor for measuring high frequency pulses because of its simplicity, linearity and electromagnetic immunity [3, 21. However, in some applications, it has some disadvantages with respect to the prototype studied. It is an intrusive transducer that affects the circuit operation and causes a voltage drop in its terminals, decreasing and damping the current. When used in high voltage systems, the resistance does not isolate the measuring equipment from the circuit under test, contributing to shunt a part of the current to the measuring system and therefore putting the personnel and equipment at risk. In this sense, if a ground fault occurs through the resistance, the measuring system is set to a high voltage with potentially serious consequences. What might be its greatest drawback is that it cannot be used in an on-line detection of high amplitude current pulses such as electrical discharges in plasmas.

Figs. 15 and 16 show two examples of pulses measured with the resistance (upper plots) and the integrator pro-

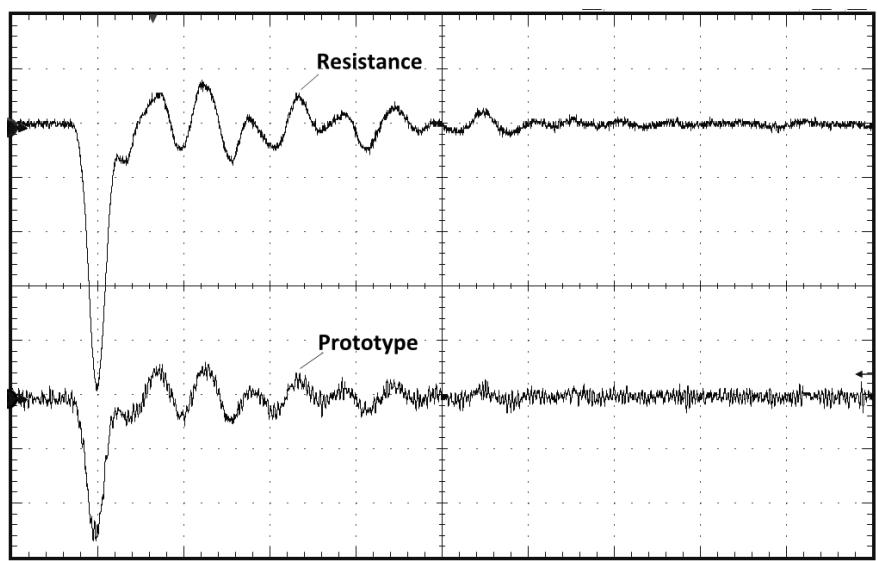

Fig. 15: Negative current pulse that corresponds to a $\mathrm{PD}$ at $2000 \mathrm{~V}$ in the polymer sheet measured with the reference resistance (upper plot) and the integrator prototype (lower plot). The vertical scales per division are 300 $\mathrm{mV}$ and $40 \mathrm{mV}$ for the resistance and the final prototype, respectively, and the time per division is $100 \mathrm{~ns}$.

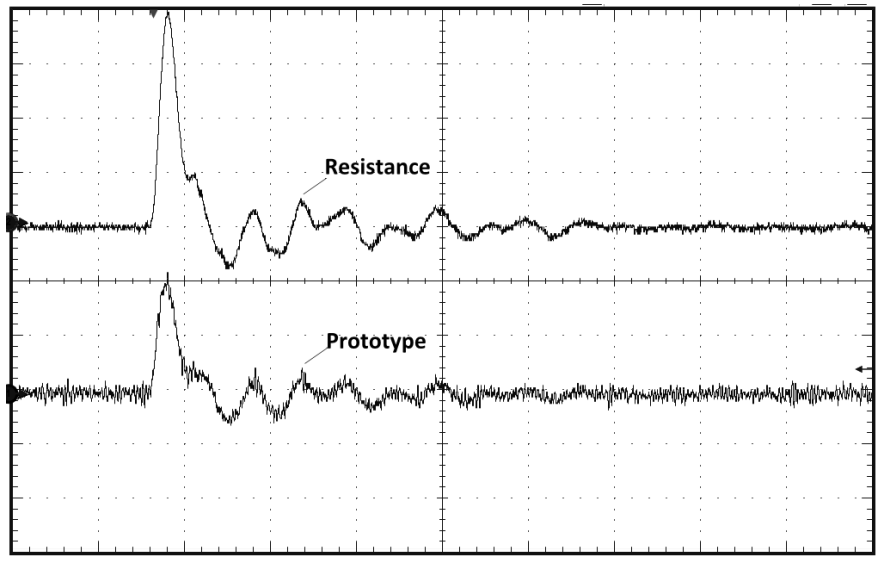

Fig. 16: Positive current pulse that corresponds to a PD at $2000 \mathrm{~V}$ in the polymer sheet measured with the reference resistance (upper plot) and the integrator prototype (lower plot). The vertical scales per division are $300 \mathrm{mV}$ and 40 $\mathrm{mV}$ for the resistance and the final prototype, respectively, and the time per division is $100 \mathrm{~ns}$.

totype (lower plots). In these cases, the vertical scales per division are $300 \mathrm{mV}$ and $40 \mathrm{mV}$ for the resistance and the final prototype, respectively, and the sampling frequency is $2.5 \mathrm{GS} / \mathrm{s}$. Focusing on the negative pulse, Fig. 15, the current through the resistance is the value of the voltage at the pulse peak divided into the parallel between the resistance $(50 \Omega)$ and the input impedance of the oscilloscope $(50 \Omega)$, yielding $-1.44 \mathrm{~V} / 25 \Omega=-57.6$ $\mathrm{mA}$. The proposed sensor reads $112 \mathrm{mV}$, so the sensitivity is $112 / 57.6 \approx 2 \mathrm{~V} / \mathrm{A}$. The same calculations can be performed in the case of the positive pulse, obtaining the same result.

It has been demonstrated that the integrator prototype has a suitable response in the range of the frequency between $1 \mathrm{MHz}$ and $50 \mathrm{MHz}$. Furthermore, it is less 


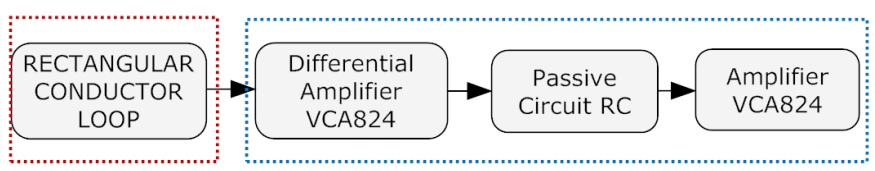

Fig. 17: The final prototype.

TABLE II: Errors of the geometrical parameters claimed by the PCB manufacturer.

\begin{tabular}{|c|c|c|}
\hline \multicolumn{3}{|c|}{ Dimensions $[\mathrm{mm}]$} \\
\hline$\delta_{l_{1}}$ & $\delta_{l_{2}}$ & $\delta_{a}$ \\
\hline 0.2 & 0.2 & 0.1 \\
\hline
\end{tabular}

expensive than an HFCT and overcomes the disadvantages of the resistive transducer in high voltage applications.

\section{Measurement Uncertainty of the Final Prototype}

Measurement uncertainty can be derived from the different stages in the measuring chain shown in Fig. 17, which can be simplified into two main blocks: the inductive loop and the integration stage.

The study of the uncertainty of the output voltage at the ends of the inductive loop is based on the mutual inductance $M$ according to (1) whereas $M$ depends on the geometry of the loop as in (2).

The lengths $l_{1}$ and $l_{2}$ and the separation distance $a$ have uncertainties induced in the manufacturing process of the $\mathrm{PCB}$, so the uncertainty in the mutual inductance should be derived from the general equation of error propagation shown in (7) 22. In this equation, $\delta_{M}$ is the absolute error of $M$ and $\delta_{l_{1}}, \delta_{l_{2}}$ and $\delta_{a}$, the absolute errors of the geometrical parameters claimed by the PCB manufacturer (see Table II). In general, track lengths have an uncertainty of $0.1 \mathrm{~mm}$ and the separation between tracks has an uncertainty of $10 \%$ of the track's width. In the calculations, a maximum error of $0.1+0.1=0.2 \mathrm{~mm}$ was used for the lengths considering that there is an uncertainty at the start and the end points of the segments. A less conservative criterium would have been the use of the quadratic sum of the uncertainties $\sqrt{0.1^{2}+0.1^{2}}=0.14$ $\mathrm{mm}$. The partial derivatives with respect to $l_{1}, l_{2}$ and $a$ are shown in (8), (9) and (10), respectively. These derivatives show the sensitivity of the mutual inductance with respect to the geometry of the sensor, so $a$ is the most critical parameter both in the design of the loop and in the uncertainty of the output voltage.

$$
\begin{aligned}
\delta_{M}=\sqrt{\left(\frac{\partial M}{\partial l_{1}} \delta_{l_{1}}\right)^{2}+\left(\frac{\partial M}{\partial l_{2}} \delta_{l_{2}}\right)^{2}+\left(\frac{\partial M}{\partial a} \delta_{a}\right)^{2}} \\
\frac{\partial M}{\partial l_{1}}=\frac{\mu_{0}}{2 \pi} \ln \frac{l_{2}+a}{a}=0.46 \mu \mathrm{H} / \mathrm{m} \\
\frac{\partial M}{\partial l_{2}}=\frac{\mu_{0}}{2 \pi} l_{1} \frac{1}{l_{2}+a}=2.4 \mu \mathrm{H} / \mathrm{m} \\
\frac{\partial M}{\partial a}=-\frac{\mu_{0}}{2 \pi} l_{1} \frac{l_{2}}{a\left(l_{2}+a\right)}=-21.2 \mu \mathrm{H} / \mathrm{m}
\end{aligned}
$$

$$
\begin{array}{r}
\delta_{M}=\sqrt{\left(91.5 \cdot 10^{-12}\right)^{2}+\left(0.48 \cdot 10^{-9}\right)^{2}+\left(-2.1 \cdot 10^{-9}\right)^{2}} \\
\cdots=2.2 \mathrm{nH} . \quad(11)
\end{array}
$$

The absolute error for $M$ is shown in (11), yielding $M=$ $54.9 \pm 2.2 \mathrm{nH}$ or $M=54.9 \mathrm{nH} \pm 4 \%$. This percentage error is also the uncertainty in the output voltage of the loop.

The following stage is the signal amplification and integration, whose uncertainty would be defined by the resistances used in the VCA 824 gain settings and the inherent gain uncertainty of the differential amplifiers.

The resistance sizes and type were 0603 and 0805 and thin film, respectively, so their relative error is as low as $0.1 \%$. The gain of the differential amplifiers is a resistances ratio $R_{F} / R_{G}, R_{F}$ being the feedback resistance and $R_{G}$ the gain resistance connected to the input of the differential amplifier. The gain uncertainty $\delta_{A R}$ due to the resistances is defined in 12, yielding $0.14 \%$.

$$
\delta_{A R}=\sqrt{\delta_{R_{F}}^{2}+\delta_{R_{G}}^{2}} .
$$

There is still the inherent uncertainty in the differential amplifier gain to consider. According to the data sheet, for a $10 \mathrm{~V} / \mathrm{V}$ gain, the error is $0.1 \mathrm{~dB}$, which corresponds to $0.12 \mathrm{~V} / \mathrm{V}$, so the relative error is $1.2 \%$. This uncertainty is notably larger than the gain uncertainty due to the resistances, so the latter is neglected and the differential amplifier gain error ends up being $\delta_{A_{1}}=1.2 \%$.

Considering the same gain error for the second amplifying stage, the total uncertainty for the differential amplifiers is obtained with 13 and gives $\delta_{A}=1.6 \%$.

$$
\delta_{A}=\sqrt{\delta_{A_{1}}^{2}+\delta_{A_{2}}^{2}} .
$$

Gathering together all the uncertainty calculations in (14), the total uncertainty in the output voltage is $\delta_{V}=$ $4.3 \%$. This value is dominated by the mutual inductance uncertainty and the distance $a$ between the loop and the primary conductor in particular. The importance of this parameter was one of the reasons for fixing it as exactly as possible on a PC-board.

$$
\delta_{V}=\sqrt{\delta_{M}^{2}+\delta_{A}^{2}}=4.3 \% .
$$

The RC integrating network does not contribute to the gain error but to the integration pole placement. Considering a percentage error of $0.1 \%$ for the resistance and $5 \%$ for the capacitance (2220 Mica), the combined error given by 15 is $5 \%$. This means that the pole is placed in $128 \pm 6 \mathrm{kHz}$ and does not really affect the measurements.

$$
\delta_{R C}=\sqrt{\delta_{R}^{2}+\delta_{C}^{2}} .
$$

Table III contains the uncertainty budget associated with the proposed sensor. In general, we may infer that $\delta_{V}$ is a result in line with the tolerance of commercial transducers. 
TABLE III: Uncertainty Budget of the Proposed Prototype

\begin{tabular}{|c|c|c|c|c|}
\hline & PARAMETER & $\begin{array}{l}\text { NUMERICAL } \\
\text { VALUE }\end{array}$ & ERROR & OBSERVATION \\
\hline \multirow[t]{2}{*}{ LOOP } & $\begin{array}{r}a \\
l_{1} \\
l_{2}\end{array}$ & $\begin{array}{l}1.016 \mathrm{~mm} \\
120 \mathrm{~mm} \\
9 \mathrm{~mm}\end{array}$ & $\begin{array}{l}10 \% \\
1,67 \% \\
2,22 \%\end{array}$ & \multirow{2}{*}{$\begin{array}{l}\text { The loop uncertainty was } \\
\text { based on } M \text { according to } \\
\text { the law of propagation of } \\
\text { uncertainty. }\end{array}$} \\
\hline & \multicolumn{3}{|c|}{ Loop Uncertainty: $\delta_{M}=4 \%$} & \\
\hline \multirow{3}{*}{$\begin{array}{l}\text { Differential } \\
\text { Amplifier }\end{array}$} & $R_{F}$ & $430 \Omega$ & $0.1 \%$ & \multirow{7}{*}{$\begin{array}{l}\text { It assumes that the } \\
\text { resistive errors are } \\
\text { negligible with respect to } \\
\text { the error given by the } \\
\text { VCA } 824 \text { specifications. }\end{array}$} \\
\hline & $\begin{array}{c}R_{G} \\
\mathrm{VCA} 824\end{array}$ & $82 \Omega$ & $\begin{array}{l}0.1 \% \\
1.2 \%\end{array}$ & \\
\hline & First Amplifie & Uncertainty: $\delta_{A}$ & $=1.2 \%$ & \\
\hline \multirow{4}{*}{$\begin{array}{l}\text { Second } \\
\text { Amplifier }\end{array}$} & $R_{F}$ & $430 \Omega$ & $0.1 \%$ & \\
\hline & $R_{G}$ & $82 \Omega$ & $0.1 \%$ & \\
\hline & VCA 824 & - & $1.2 \%$ & \\
\hline & \multicolumn{3}{|c|}{ Second Amplifier Uncertainty: $\delta_{A_{2}}=1.2 \%$} & \\
\hline \multicolumn{5}{|c|}{ The Prototype Uncertainty: $\delta_{V}=4.3 \%$} \\
\hline
\end{tabular}

\section{CONClusion}

An inexpensive and simple inductive probe based on the Faraday Law and used for measuring fast current pulses, was presented showing good results. The detection of partial discharges was the application chosen for its validation. The measurements were also taken with two sensors: a high frequency current transformer and a resistance, the former to verify the sensor response and the latter to estimate the current value and the sensitivity of the final prototype. Initially, we have found a linear response up to approximately $30 \mathrm{MHz}$, good sensitivity and complete repeatability in the measurements. However, with the implementation of the integrator in the sensor, the bandwidth is extended up to $50 \mathrm{MHz}$. Furthermore, the experimental validation demonstrated that the final prototype can be considered a suitable transducer for measuring high frequency pulses with an uncertainty similar to other commercial devices. Thus, we have highlighted some remarkable advantages such as the inductive coupling that prevents direct connection to the circuit under test and permits the protection of the measuring system and, finally, the possibility of its future application in on-line detection and its possible use as a reference transducer for other sensors.

\section{ACKNOWLEDGMENTS}

This research was supported by the Spanish Science and Technology Ministry under contract DPI 2009-14628-C0302. Tests were done in the High Voltage Research and Test Laboratory of Universidad Carlos III de Madrid and the Electromagnetic Compatibility Laboratory of Università di Pisa, DSEA. Also, the authors appreciate the help of Ernesto García Ares, Engineer in the technical office of Universidad Carlos III de Madrid in the design and implementation of the integrator prototype.

\section{REFERENCES}

[1] M. S. Naidu and V. Kamaraju, High Voltage Engineering. New Delhi: MacGraw-Hill, 2009.

[2] K. L. Kaiser, Electromagnetic Compatibility Handbook. U.S.A.: CRC Press, 2005.

[3] J. G. Webster, The Measurement, Instrumentation and Sensors Handbook. U.S.A.: CRC Press, 1999.
[4] D. C. Smith, High Frequency Measurements and Noise in Electronic Circuits. New York: Van Nostrand Reinhold, 1993.

[5] M. Muhr and R. Schwarz, "Partial discharge measurement as a diagnostic tool for HV-Equipments," in Properties and applications of Dielectric Materials, 2006. 8th International Conference on, pp. 195-198, 2006.

[6] S. Boggs and G. Stone, "Fundamental limitations in the measurement of corona and partial discharge," Electrical Insulation, IEEE Transactions on, vol. EI-17, no. 2, pp. 143-150, 1982.

[7] F. H. Kreuger, Partial Discharge Detection in High-Voltage Equipment. London: Butterworths, 1989.

[8] Bartnikas and McMahon, Engineering Dielectrics, Volume I. Corona Measurements and Interpretation. Philadelphia: ASTM, 1979.

[9] R. Tallam, S. B. Lee, G. Stone, G. Kliman, J. Yoo, T. Habetler, and R. Harley, "A survey of methods for detection of StatorRelated faults in induction machines," Industry Applications, IEEE Transactions on, vol. 43, no. 4, pp. 920-933, 2007.

[10] IEC 60270, High Voltage Test Techniques. Partial Discharge Measurements, 3.0 ed., 2000.

[11] G. Robles, J.M.Martínez, M. Rojas, and J.Sanz, "Inductively coupled probe for the measurement of partial discharges," Review of Scientific Instruments, vol. 79, no. 5, 2008.

[12] G. Robles, J.M.Martínez, M. Rojas, and J.Sanz, "Inductive sensor for measuring high frequency partial discharges within electrical insulation," IEEE Transactions on Instrumentation and Measurement, vol. 58, no. 11, 2009.

[13] S. Ramo, J. Whinnery, and T. V. Duzer, Fields and Waves in Communication Electronics. New York: John Wiley and sons, 1993.

[14] F. W. Grover, Inductance Calculations. New York: Dover Phoenix, 1973.

[15] A. Cavallini, G. Montanari, A. Contin, and F. Pulletti, "A new approach to the diagnosis of solid insulation systems based on PD signal inference," IEEE Electrical Insulation Magazine, vol. 19, pp. 22-30, Mar-Apr 2003.

[16] T. Instruments, "Wideband $420 \mathrm{mhz}, 40 \mathrm{db}$ gain adjust range, linear in v/v variable gain amplifier vca824." Datasheet.

[17] E. Gulski, S. Meijer, M. Muhr, T. Strehl, and S. Tenbolen, "Guidelines for un-conventional partial discharge measurements." Cigré, 2006.

[18] S. H. Kan, Metric and models in software quality engineering. Boston: Pearson Education, Inc, 2nd ed., 2003.

[19] M. Rojas, G. Robles, B. Tellini, C. Zappacosta, J. Martínez, and J. Sanz, "An inductive transducer for the measurement of high frequency pulses with applicability in the detection of partial discharge," in Instrumentation and Measurement Technology Conference Proceedings, 2010. IMTC 2010. IEEE, pp. 375-379, 2010.

[20] T. E. Corporations, "Bnc plug termination 50 ohms." Datasheet.

[21] S. A. Dyer, Survey of instrumentation and measurement. New York: John Wiley and sons, 2001.

[22] NIST, Guidelines for Evaluating and Expressing the Uncertainty of NIST Measurements Results, 1994. 